\title{
Herpes Zoster, Unusual Skin Presentation as Part of Wegener's Granulomatosis: A Case Report
}

\author{
Abbas Rasi, ${ }^{1}$ Abbas Zamanian, ${ }^{1}$ Nasrin Shayanfar, ${ }^{2}$ Elham Behrangi, ${ }^{1}$ and Zahra Azizian ${ }^{1,}{ }^{*}$ \\ ${ }^{1}$ Department of Dermatology, Rasoul Akram Hospital, Iran University of Medical Sciences, Tehran, IR Iran \\ ${ }^{2}$ Department of Pathology, Rasoul Akram Hospital, Iran University of Medical Sciences, Tehran, IR Iran \\ "Corresponding author: Zahra Azizian, Department of Dermatology, Rasoul Akram Hospital, Iran university of Medical Sciences, Tehran, IR Iran. Tel: +98-2177618221, E-mail: \\ azizian_z@yahoo.com
}

Received 2015 March 03; Revised 2015 June 10; Accepted 2015 June 10.

\begin{abstract}
Introduction: Wegener's granulomatosis is a lethal disease with granulomatous inflammation and necrotizing vasculitis. Case Presentation: The case was a 63-year-old female with a history of Wegener's granulomatosis presented with erythema of the arm within which there was a crusted or ulcerated lesion surrounded by small vesicles that was confirmed as herpes simplex infection.

Conclusions: Current standard of care for treatment of Wegener's regimens are related to opportunistic infections. Wegener's granulomatosis is a vasculitis that the presence of cutaneous presentation of herpes zoster would be explained by the modulation of immunity.
\end{abstract}

Keywords: Wegner Granulomatosis, Vasculitis, Disease

\section{Introduction}

Wegener's granulomatosis (WG) is a partially rare and lethal disease, characterized by granulomatous inflammation, and necrotizing vasculitis of small and medium size vessels (1). Its cause is not known. Multiple organs are involved, particularly the upper respiratory tract, the lungs, the kidney and the eyes $(2,3)$. It affects both males and females. It can begin at any age with a peak during the $5^{\text {th }}$ and $6^{\text {th }}$ decades of life with initial symptoms being nonspecific. They mostly include symptoms in the upper respiratory tract, weakness, fatigue, arthralgia and fever without an infectious cause.

Glomerulonephritis (GN) is a common cause of morbidity and mortality. Its expression can be indolent or more frequently, aggressive. Urine analysis has indicated proteinuria and an increased level of urea and serum creatinine. Renal biopsy of patients with Wegener's Granulomatosis has mostly shown modifications of necrotic, segmentary and focal GN and sometimes in the absence of significant deposits of immunoglobulin and complement and more rarely diffuse necrotic GN with different proliferating modifications.

Skin involvement has been seen in nearly $50 \%$ of patients. The initial manifestation of the disease may include deep inflammatory cutaneous plaques, acute or indolent. Other manifestations are palpable purpura, pyoderma-like ulcerations, gingival hyperplasia, inflammatory papule, small ulcers, panniculitis and subcutaneous nodules (4). Generally, the activity of cutaneous affection is related to the systemic disease. Although the histopathology for WG is not pathognomonic for cutaneous involvement.

\section{Case Presentation}

The case was a 63-year-old female who had presented the first symptoms of Wegener's disease four years ago: sinusitis and prolonged fever. The otorhinolaryngologic examination rendered evident lesions of the upper respiratory tract. The performance of antineutrophil cytoplasmic antibodies (ANCA) was positive with an initial titre of 1/170 with perinuclear pattern in immunofluorescence. The diagnosis of Wegener's granulomatosis was established and the patient was treated with prednisolone and cyclophosphamide. The disease improvement was great obtaining a high improvement in biological activity markers (PCANCA decreased to 1/20). Recently, the patient was presented to the emergency department with lower extremities and palpebral edema. She also complained of the appearance of a mild painful ulcer with an erythematous background, located on the middle section of her right arm, which had appeared seven days prior to her referral.

On examination, there was erythema of the arm within which there was a crusted or ulcerated lesion surrounded by small vesicles (Figure 1). Its appearance looked like vas- 
culitis so the patient underwent a punch biopsy. The diagnosis of herpes zoster was confirmed by the biopsy. In the biopsy, ballooning degeneration and herpetic cytopathic changes were evident (Figure 2). Acantholysis or multinucleated keratinocytes formation were obvious (Figure 3). A treatment with acyclovir $10 \mathrm{mg} / \mathrm{kg}$ was set for seven days. After two weeks the herpetic lesion evolved greatly (Figure 4). Laboratory tests indicated anemia, increased rate of blood segmentation, proteinuria, low albumin and a high level of serum creatinine. A renal biopsy puncture showed lesions of diffused extra-capillary glomerulonephritis with sclerosis. The patient was followed for about one year. There was no recurrence of herpes zoster, yet two flares of renal disease with rise of creatinine occurred.

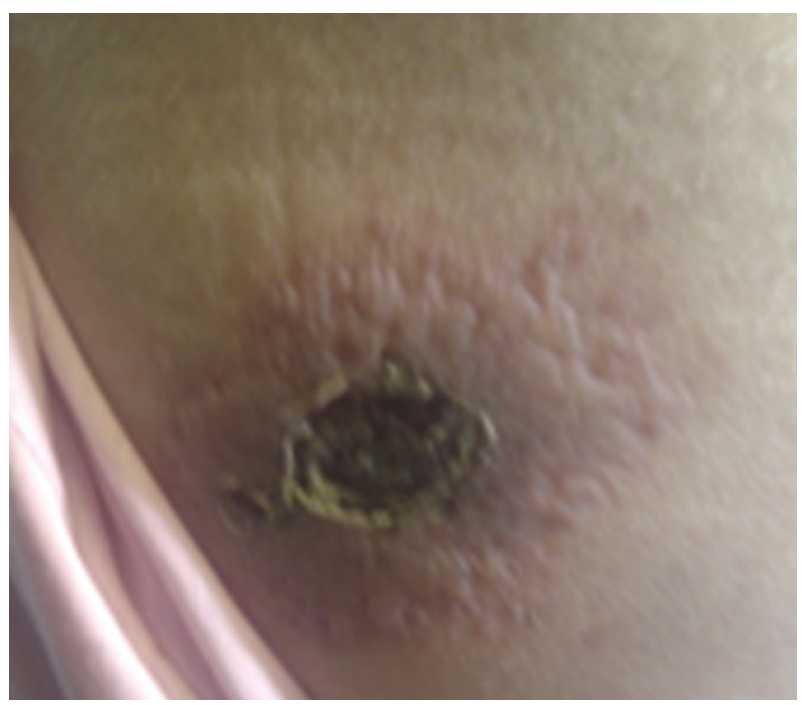

Figure 1. Necrotic, Crusted Lesion on the Right Arm

\section{Discussion}

We presented a more unusual case of WG, who had indications of infection of the upper respiratory tract, kidneys and skin yet in a totally uncharacteristic way. Our case was rare due to herpes infection one week prior to disease recurrence and also strange presentation of herpes zoster, which resembled vasculitis. This report could serve as a guide for physicians, informing them that herpes zoster infection could be a prior presentation before WG recurrence and also skin presentation of herpes zoster in Wegener's granulomatosis could mimic other diseases. The presence of cutaneous presentation of herpes zoster would be explained by the modulation of immunity. Herpes zoster is a painful neurocutaneous disease caused by

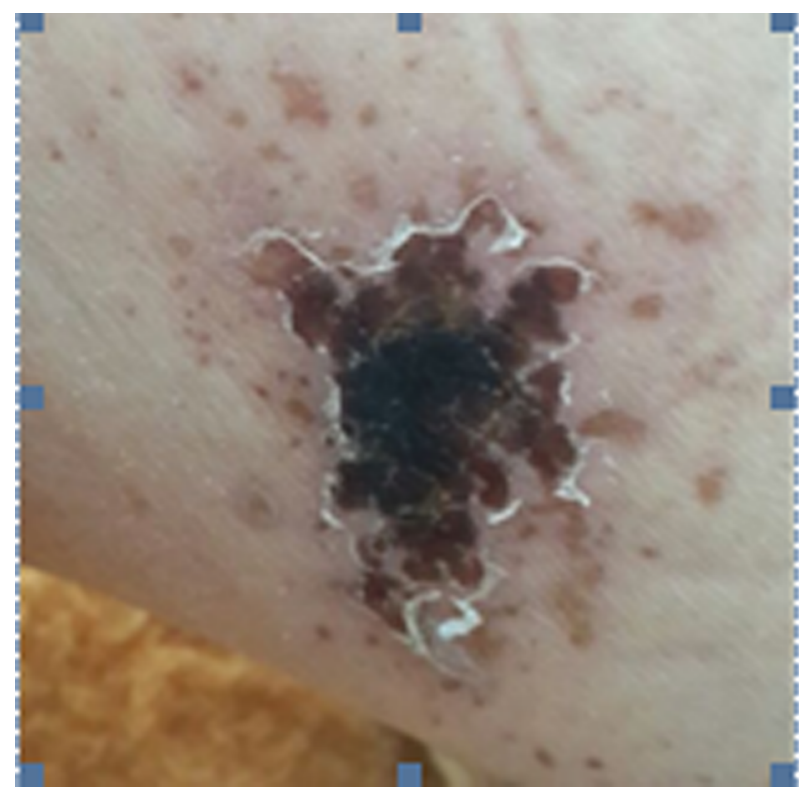

Figure 2. Two Weeks After Treatment

Figure 3. Histopathology of the Skin Lesion

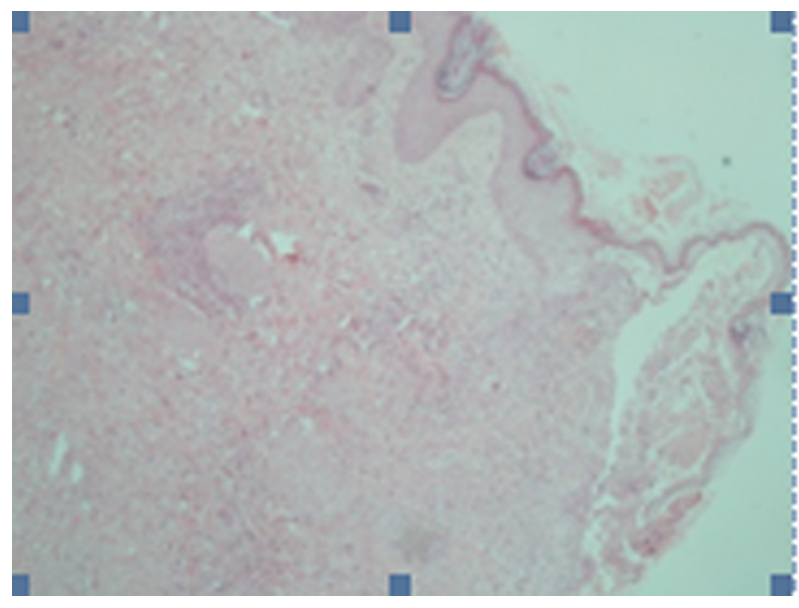

The image indicates intraepidermal vesicle and perivascular and diffuse infiltration of lymphocytes.

reactivation of Varicella zoster virus (5). A variety of immunocompromised patients are known to be at increased risk of herpes zoster $(6,7)$. Wegener's granulomatosis (WG) is a type of systemic vasculitis characterized by necrotizing granulomatous inflammation (8). Immunosuppressive regimens, especially high doses of glucocorticoids and cyclophosphamides, induce remission in about $80 \%$ to $90 \%$ of patients $(9,10)$; unfortunately, disease flares following remission are common. Current standard of 
Figure 4. Herpetic Cytopathic Changes Such as Pale Keratinocytes With Margination of Chromatin at the Edge of Nucleus and Intranuclear Inclusions

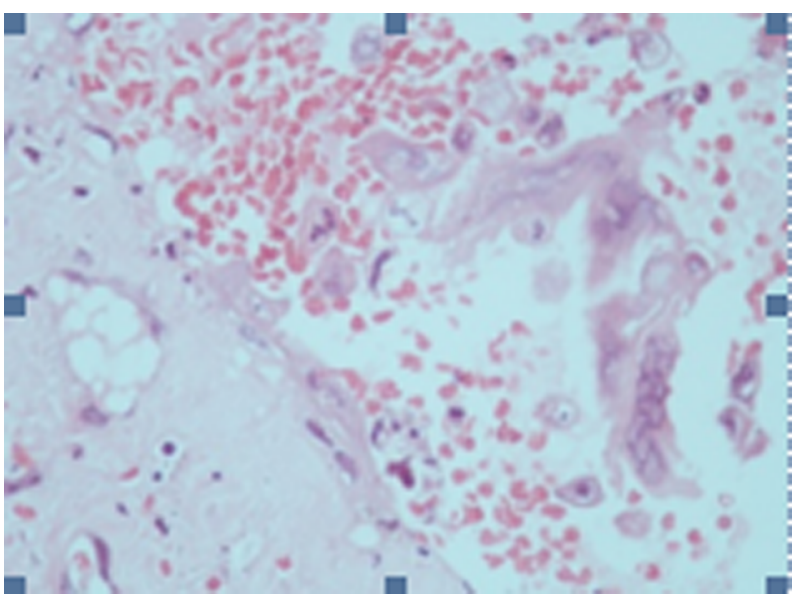

The extravasation of erythrocytes is obvious.

care treatments are related to opportunistic infections (9). Cupps et al. reported on the frequent occurrence of herpes zoster among Wegener's granulomatosis, with the Fauci and Wolff regimen, which includes "initially high doses of glucocorticoids and daily cyclophosphamide". Recently, shorter courses of cyclophosphamide are employed for remission reduction; however, the risk of occurrence increases. Renal dysfunction and female sex were consistently strong risk factors for herpes zoster in a study by Wung et al. (11). Thus, it is recommended to prevent herpes zoster in patients on treatment for immune-mediated diseases. Another special feature of our case was the discovery of anti-cytoplasmic neutrophilic antibodies namely anti-peroxidase (mPO3) antibodies with perinuclear pattern (P-ANCA) by the immunofluorescence technique. As it has been documented anti-protein 3 antibodies with cytoplasmic antineutrophil cytoplasmic antibod (CANCA) are a characteristic of WG. However, anti-peroxidase antibodies in 5 to $10 \%$ of patients with WG are associated with P-
ANCA. Another special character of our case was recovery for about four years since the initiation of disease showing good prognosis; however, recurrence with renal involvement indicating a bad prognosis.

\section{Footnote}

Authors' Contribution: Zahra Azizian: data collection; Elham Behrangi: prepairing articles; Abbas Zamanian: preparation of the article; Nasrin Shayanfar: pathology preparation and Abbas Rasi: data collection.

\section{References}

1. Feier V. Dermatovenerologic. Timisoara: Editura Amarcord; 1998. pp. 17-73.

2. Harman LE, Margo CE. Wegener's Granulomatosis. Surv Ophthalmol. 1998;42:458-80.

3. Pakrou N, Selva D, Leibovitch I, editors. Wegener's granulomatosis: ophthalmic manifestations and management. Seminars in arthritis and rheumatism. 2006; Elsevier; pp. 284-92.

4. Nolle B. Anticytoplasmic Autoantibodies: Their Immunodiagnostic Value in Wegener Granulomatosis. Annal Inter Med. 1989;111(1):28. doi: 10.7326/0003-4819-111-1-28.

5. Gnann JJ, Whitley RJ. Clinical practice. Herpes zoster. N Engl J Med. 2002;347(5):340-6. doi:10.1056/NEJMcp013211. [PubMed: 12151472].

6. Schuchter LM, Wingard JR, Piantadosi S, Burns WH, Santos GW, Saral R. Herpes zoster infection after autologous bone marrow transplantation. Blood. 1989;74(4):1424-7.

7. Buchbinder SP, Katz MH, Hessol NA, Liu JY, O’Malley PM, Underwood $\mathrm{R}$, et al. Herpes zoster and human immunodeficiency virus infection. J Infect Dis. 1992;166(5):1153-6. [PubMed: 1308664].

8. Hoffman GS, Kerr GS, Leavitt RY, Hallahan CW, Lebovics RS, Travis WD, et al. Wegener granulomatosis: an analysis of 158 patients. Ann Intern Med. 1992;116(6):488-98. [PubMed: 1739240].

9. Wegener's Granulomatosis Etanercept Trial Research G. Etanercept plus standard therapy for Wegener's granulomatosis. $N$ Engl J Med. 2005;352(4):351-61. doi:10.1056/NEJMoa041884. [PubMed: 15673801].

10. Reinhold-Keller E, Beuge N, Latza U, de Groot K, Rudert H, Nolle B, et al. An interdisciplinary approach to the care of patients with wegnergranulomatosis: long-term outcome in 155 patients. Arthritis Rheum. 2000;43(5):1021-32.

11. Wung PK, Holbrook JT, Hoffman GS, Tibbs AK, Specks U, Min YI, et al. Herpes Zoster in immunocompromised patients incidence, timing and risk factors. Am J Med. 2005;118(12):1416. 